\title{
Performance Evaluation and Fluid Flow Analysis in Low Flow Stages of Industrial Centrifugal Compressor
}

YURI I. BIBA*, DAVID A. NYE, and ZHEJI LIU

Turbo Products Division Dresser-Rand Company, Olean, NY 14760, USA
A comparative study of the flow field and performance of centrifugal compressor stages is presented for low volume flows and high-pressure applications. Two different impeller designs and stage configurations are considered and modeled using commercial CFD codes. Internal stage designs are evaluated by qualitative and quantitative flow analysis with the goal being to obtain more efficient stages. The resulting improved configurations are implemented into one of DresserRand compressors. Computational and experimental results are discussed and conclusions are made regarding the existing model as well as future improvements both in modeling and design concept.

Keywords: Centrifugal compressor; Stage; Impeller; Performance; CFD; Low flow; Efficiency; Test rig

Centrifugal compressor impellers for low flow coefficients $\Phi$ are subjects of specific interest due to their application in process industry and gas injection markets. In these applications such impellers are often used in last stages at high-pressure levels.

A systematic study of low flow compressor stages has been presented by Casey et al. (1990). It is noticed that a simple dissipation loss model was unable to predict, with any certainty, which of the impellers of different design would have the best performance. Extensive experimental studies for all impellers are necessary to identify the most efficient one.

Impeller maximum efficiency drops rapidly with the decrease of design volume flow (Balje, 1981) making it very

\footnotetext{
Received 23 January 2001; in final form 29 January 2001

*Corresponding author. Tel.: (716)375-3000, Fax: (716)375-3178. E-mail: yuri_i_biba@dresser-rand.com
}

difficult to improve stage efficiency for lower flow values. That general trend has not changed with years of extensive development efforts by major turbomachinery manufacturers (Dalbert et al., 1999). Dresser-Rand has been using CFD to design new, more efficient, families of low flow impellers to apply in last stages of high pressure compressors.

Recent progress in Computational Fluid Dynamics (CFD) code development and decreased computing cost make more detailed computational analysis possible. At the same time, experimental testing remains very expensive and will likely need to be reduced. CFD modeling, or a Virtual Test Rig (VTR) approach is routinely used while experimental testing remains a final verification tool. Dresser-Rand has implemented the VTR concept since the company began its use of CFD software. CFD has been routinely used to compare analytical predictions with test results to augment test data and validate predictions (Sorokes and Koch, 1996).

Many issues need to be addressed before a sufficient level of confidence is gained to assess performance change via CFD studies. Part of this responsibility is certainly part of the VTR study, where the effects of grid density, windage, leakage, surface roughness, frame size, etc. will need to be addressed. However, full confidence can really only be gained through calibration and comparison with test data. This test data can originate from extended scope instrumentation on production rigs or improved Single Stage Test Rig (SSTR) testing. While the VTR is being developed the new stages can be optimized using CFD to assess additional design improvements.

\section{BACKGROUND}

It was decided to implement a new design for three last stages of a multistage high-pressure barrel compressor. Flow coefficients $\Phi$ for these stages are $0.0146,0.0117$ and 0.0097 , respectively. These three stages (Figure 1) were 


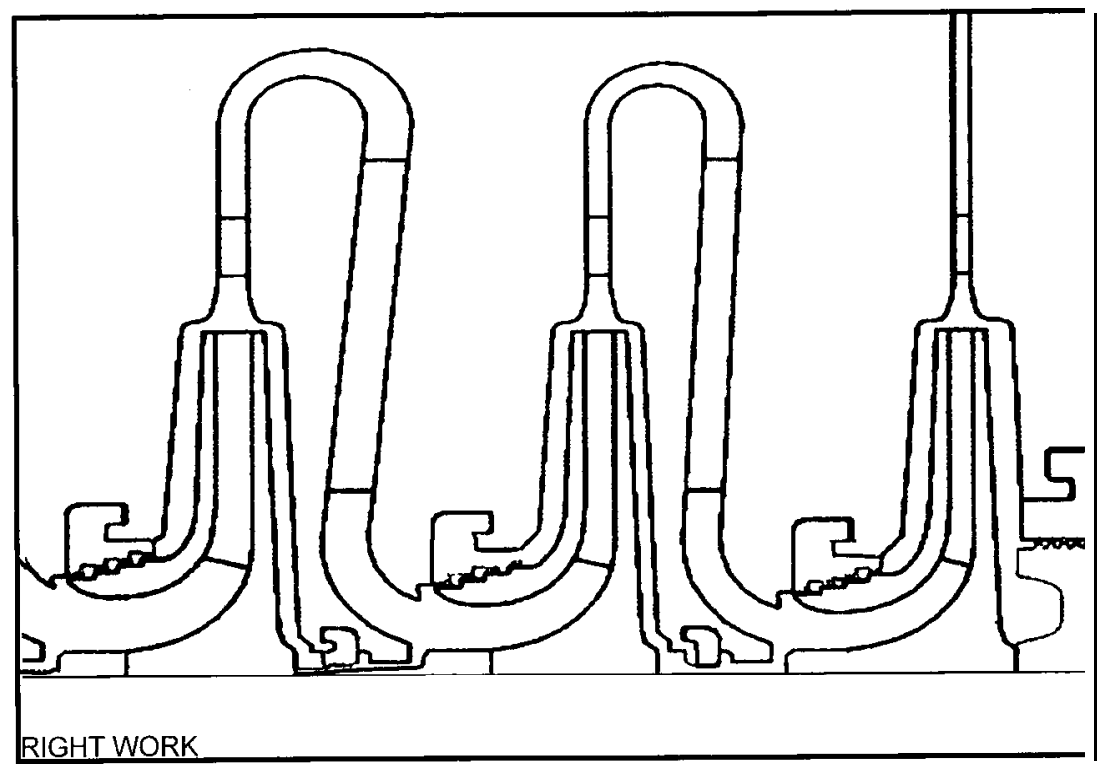

FIGURE 1 Cross section of last three stages of high pressure centrifugal compressor.

selected to replace original impellers that are characterized by relatively small backsweep and narrow flow passages.

The existing designs are replaced by the new impellers with wider passages and a significantly larger blade backsweep angle. The intent of the design was to reduce flow velocity and loading throughout the stage, make the impeller exit flow more uniform to reduce stage losses and produce slightly less head than the original design.

Preparatory study was performed to determine the optimum diffuser size. This study used Dresser-Rand's proprietary semi-empirical, one-dimensional analytical code STGPERF. A number of cases were run on STGPERF to investigate the influence of the diffuser radius ratio. This ratio was varied between 1.25 and 1.49 for stages with flow coefficients from 0.0032 to 0.0275 . Efficiency plots are shown in Figures $2 \mathrm{a}-2 \mathrm{~d}$. The results showed that for a stage $\Phi=0.0275$, the peak efficiency was achieved at the larger diffuser radius ratio. For the lowest flow stage, peak efficiency was achieved at the lower value of diffuser radius ratio, with indications that even lower values of radius ratio may be beneficial. For a stage $\Phi=0.0069$ STGPERF predicts a neutral influence of diffuser radius ratio on peak efficiency.

The conclusion drawn from the investigation is that higher flow stages require larger radius ratio vaneless diffusers than smaller flow stages. The positive influence of
Stage Performance: $\Phi=0.0275$, D5/D2=var

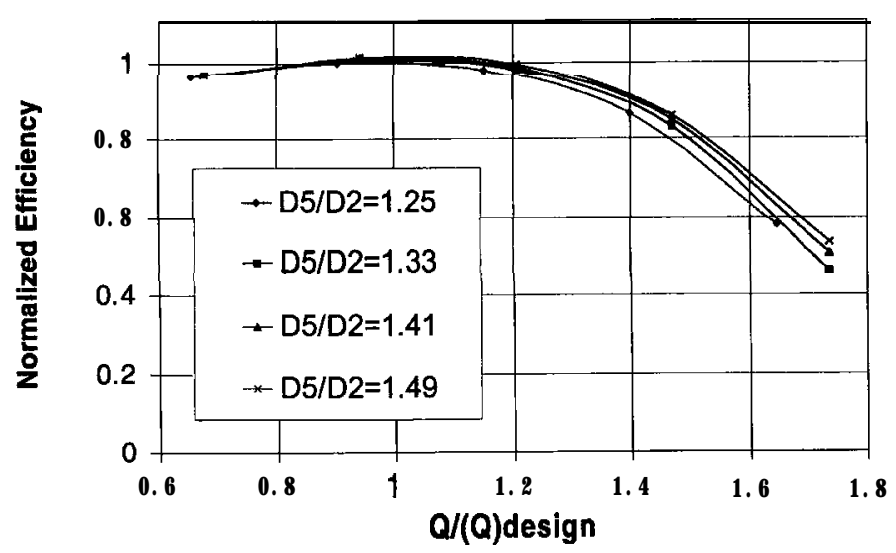

FIGURE 2a Stage performance at $\Phi=0.0275$ and various $D_{5} / D_{2}$ calculated by STGPERF.

\section{$\Phi=0.0134, D 5 / D 2=v a r$}

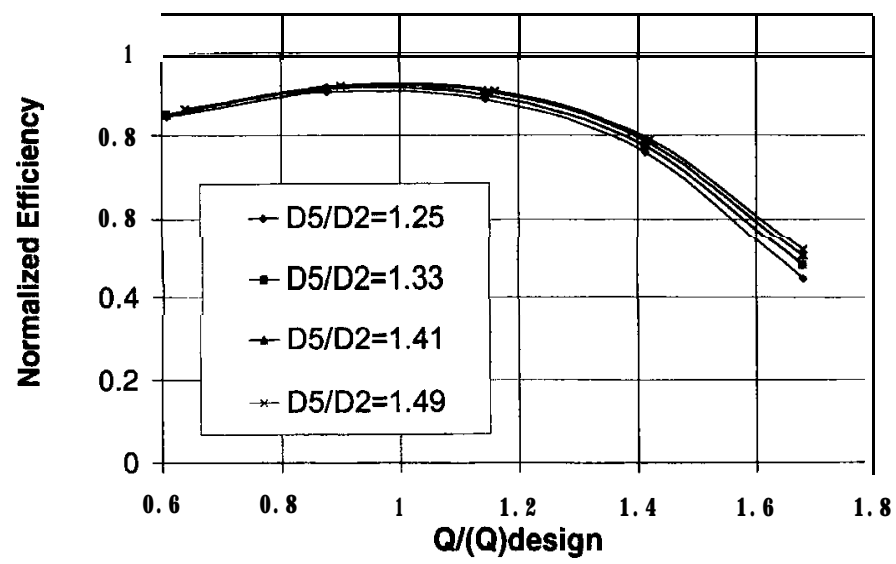

FIGURE 2b Stage performance at $\Phi=0.0134$ and various $D_{5} / D_{2}$ calculated by STGPERF. 


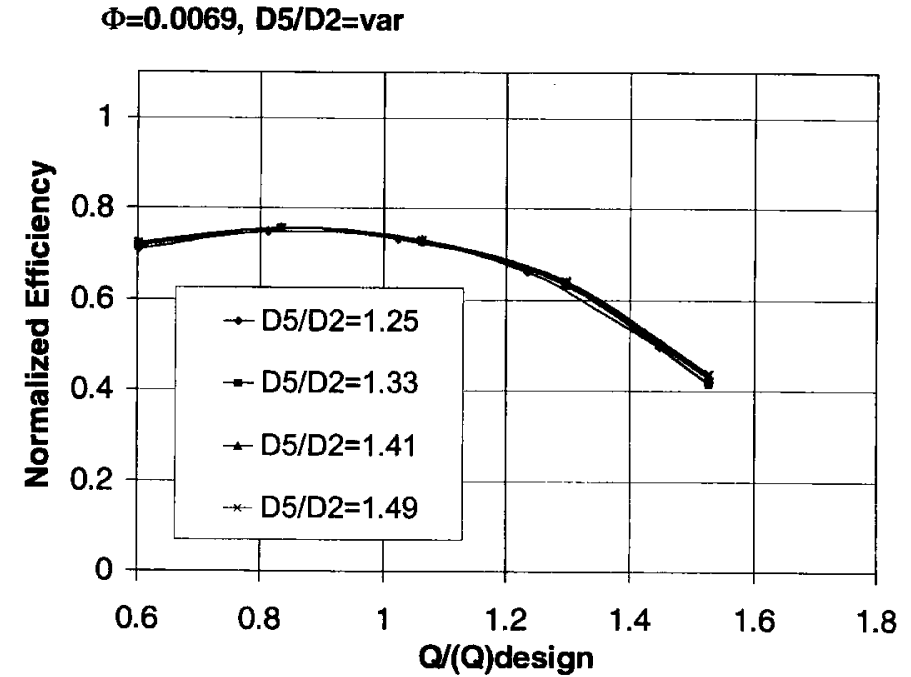

FIGURE 2c Stage performance at $\Phi=0.0069$ and various $D_{5} / D_{2}$ calculated by STGPERF.

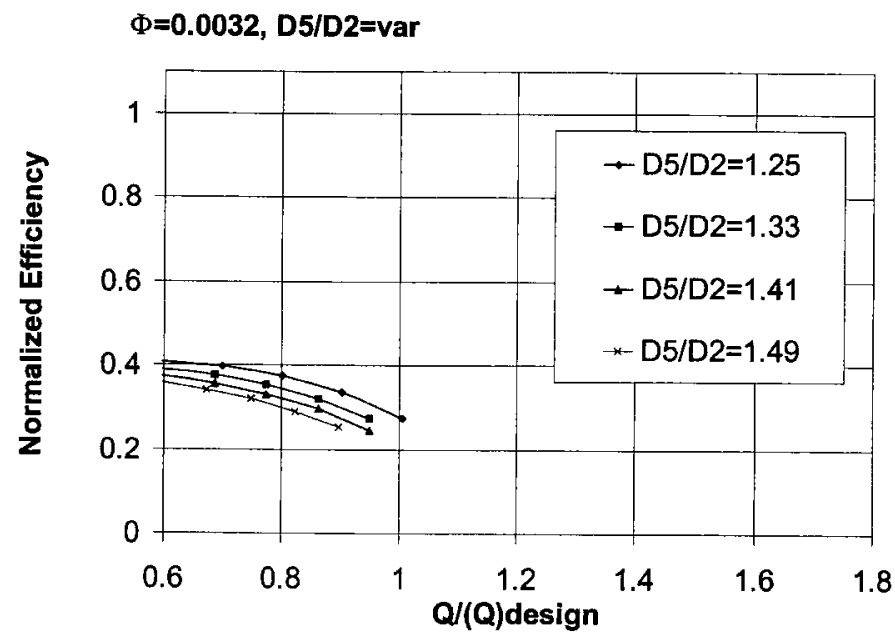

FIGURE 2d Stage performance at $\Phi=0.0032$ and various $D_{5} / D_{2}$ calculated by STGPERF.

vaned diffuser systems in lower specific flow stages does not change that conclusion; in fact it may expand this conclusion. A vaned diffuser tends to move the stage with neutral influence of diffuser radius ratio to a larger $\Phi$ value and also tends to move the minimum diffuser ratio to a lower value.

As in the existing design, an impeller with a flow coefficient 0.0275 is chosen as the parent impeller of the whole family. Each impeller with a flow coefficient less than 0.0275 (but greater that 0.0077 ) can be obtained from the parent impeller by a proper meridional contour cut. The parent impeller is included in the analysis because it is the only low flow wheel of existing design that had been tested in the SSTR until this time.
The main focus of the current work revolves around past and present stage configurations in a CFD study comparing performance of the original and new stages. The operating conditions chosen are for a single wheel speed under typical SSTR conditions: inlet pressure 30 psia, inlet temperature $100 \mathrm{deg}$. F and nitrogen as the working gas. Description of the Single Stage Test Rig can be found in the work of Sorokes and Welch (1992).

The stages compared have significantly different stationary component configurations, which are not necessarily optimum. The two fundamental differences that should be noted in this discussion are the shorter radius ratio used in the new design versus a larger radius ratio used as the current standard. In addition, due to the high backsweep used in the new impeller designs Low Solidity Diffusers (LSD) are required, whereas the old design is better served using a vaneless diffuser. Therefore the new and old low flow configurations should be compared as a stage for any proposed adjustments to overall compressor performance.

It must be noted that the CFD results should only be compared directly with other CFD results, since the modeling does not completely capture all physical reality (disk friction, leakage, etc.).

\section{CFD ANALYSIS}

Obtained flow fields are analyzed to identify possible flow separation or other specific areas of losses (like flow nonuniformity) that may cause efficiency decrease or insufficient head rise.

The stage grid consists of four parts: vaneless radial inlet region (not shown), impeller, LSD including one-half of the return bend and return channel with the remaining portion of the return bend, as shown in Figure 3.

A commercial CFD code CFX-Tascflow ${ }^{\circledR}$, a product of AEA Technology Engineering Software, was used for the stage analysis. Three "frozen rotor" interfaces were applied to attach grid regions; the total number of nodes is approximately 150,000 . One blade passage was considered for each component, with circumferentially periodic boundary conditions. Mass flow boundary was utilized at the stage outlet while averaged flow angle and stagnation pressure and temperature were imposed at the inlet.

For the parent impeller stage $(\Phi=0.0275)$ at design flow, separation is not apparent in the stationary components or impeller (Figure 4). Flow velocity remains relatively high in the inlet region due to leading edge incidence effects and blade thickness blockage. However, the relative Mach number increase is not as high as that observed in the old impeller (Figure 5). Impeller loading decreases around trailing edge, thus minimizing the wake originating from the suction side of the blade, and making the flow field 


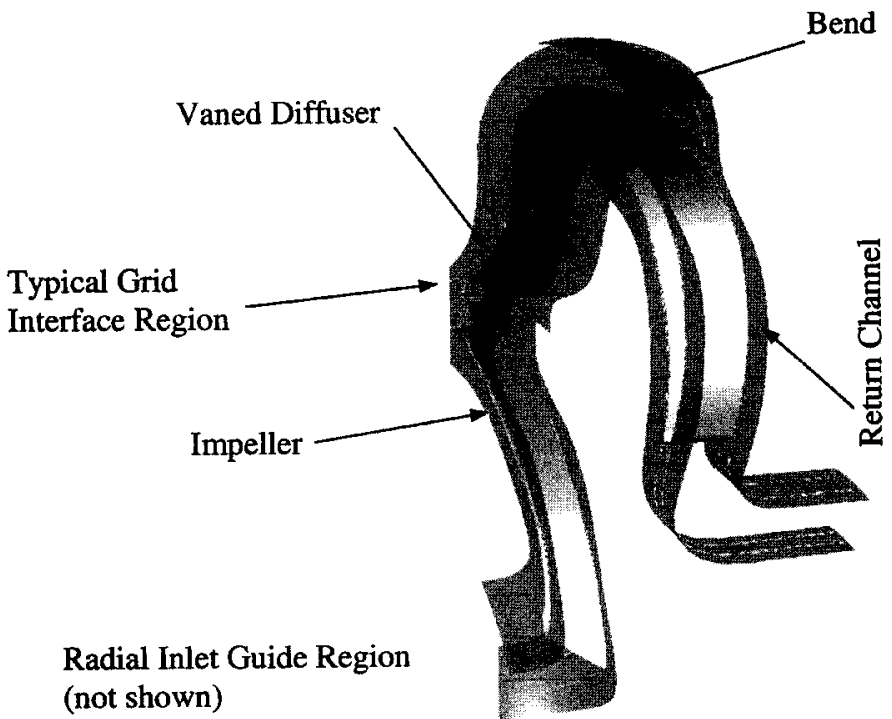

FIGURE 3 Typical CFD grid arrangement.

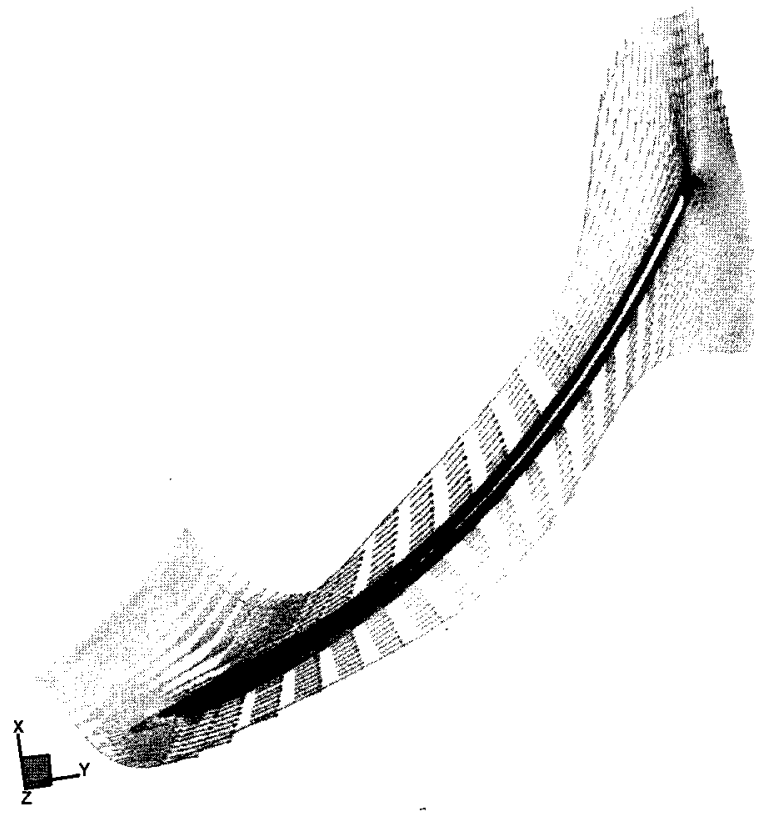

FIGURE 4 Velocity vectors colored by Mach number at design flow, $\Phi=0.0275$, new design (See Colour Plate at back of issue.).

behind the impeller more uniform. Most of the head is generated by approximately $60 \%$ of the blade in the midpassage region.

At $78 \%$ mass flow, no recirculation zones were found in the impeller. A low-energy region, mainly near the shroud surface, occurred at the suction side midway through the blade passage, but the high exit backsweep does not allow the wake to spread.

A small local recirculation zone occurs near the hub at the trailing edge of suction side of the LSD vane, but the

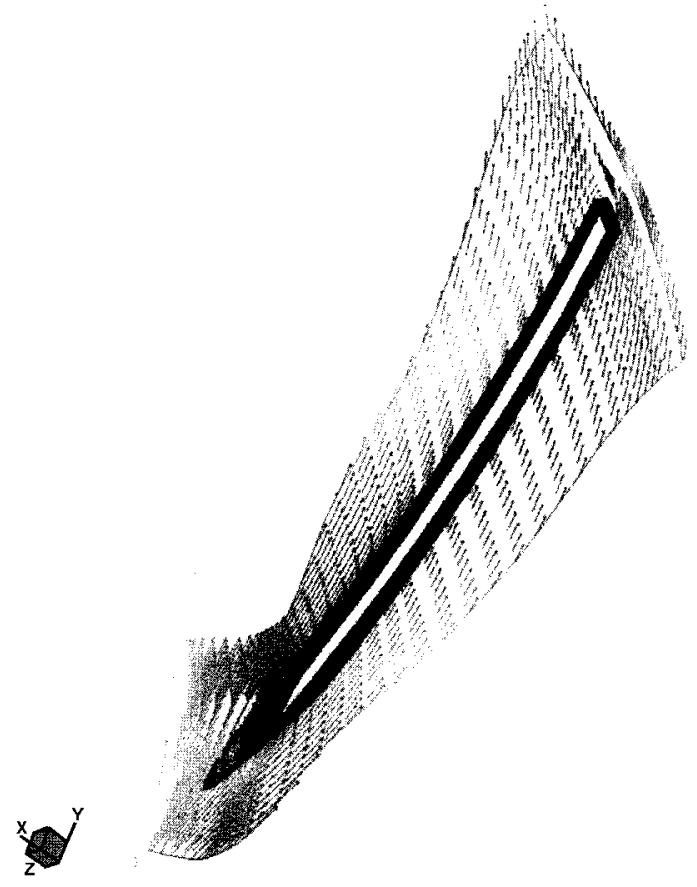

FIGURE 5 Velocity vectors colored by Mach number at design flow, $\Phi=0.0275$, old design (See Colour Plate at back of issue.).

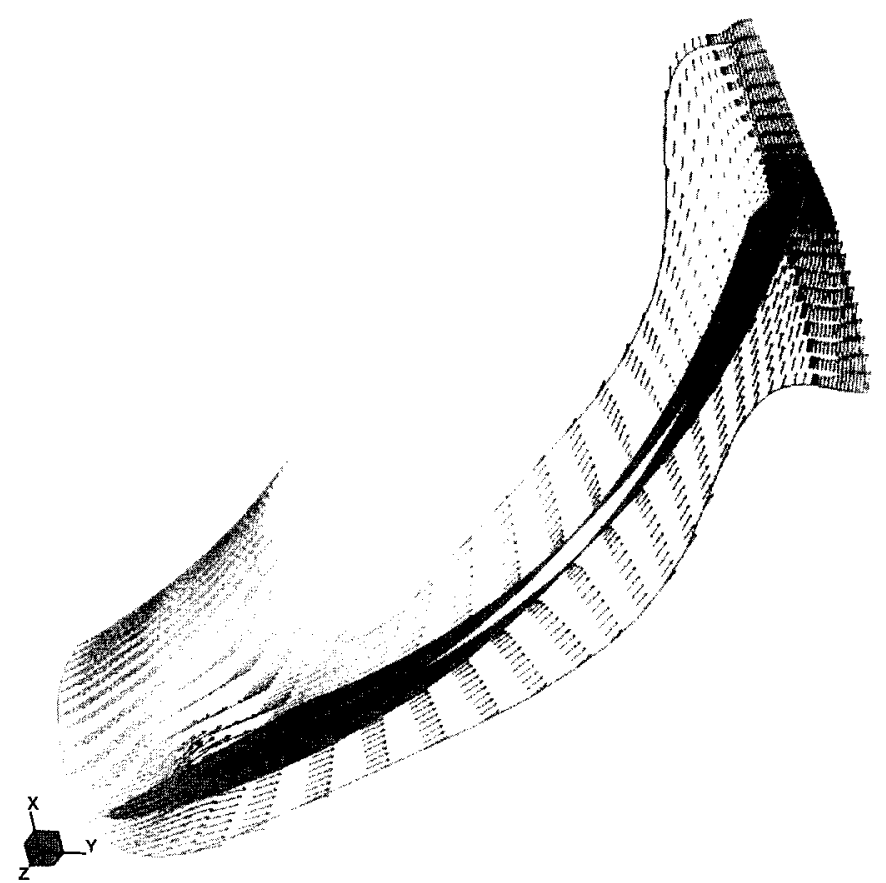

FIGURE 6 Velocity vectors colored by Mach number at $62 \%$ of design flow, $\Phi=0.0275$, new design (See Colour Plate at back of issue.).

wake is relatively small due to the higher velocity flow coming from the pressure side.

At even lower mass flow ( $62 \%$ of design value), a small separation zone with a recirculation was found at the hub-suction corner of the impeller blade passage (Figure 6). 
High velocities were observed at the impeller inlet on the suction side of the blade, resulting from incidence and flow turning around the leading edge. In the mid and exit part of the blade passage a low-energy zone can be seen at the suction side, but without separation.

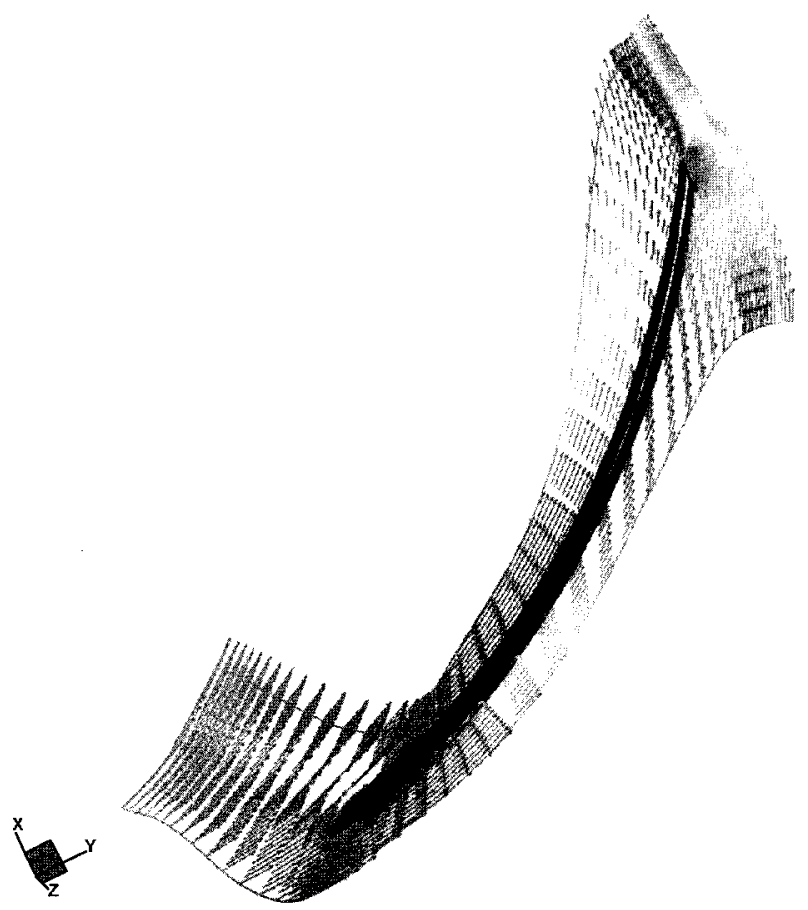

FIGURE 7 Velocity vectors colored by Mach number at design flow, $\Phi=0.0117$, new design (See Colour Plate at back of issue.).

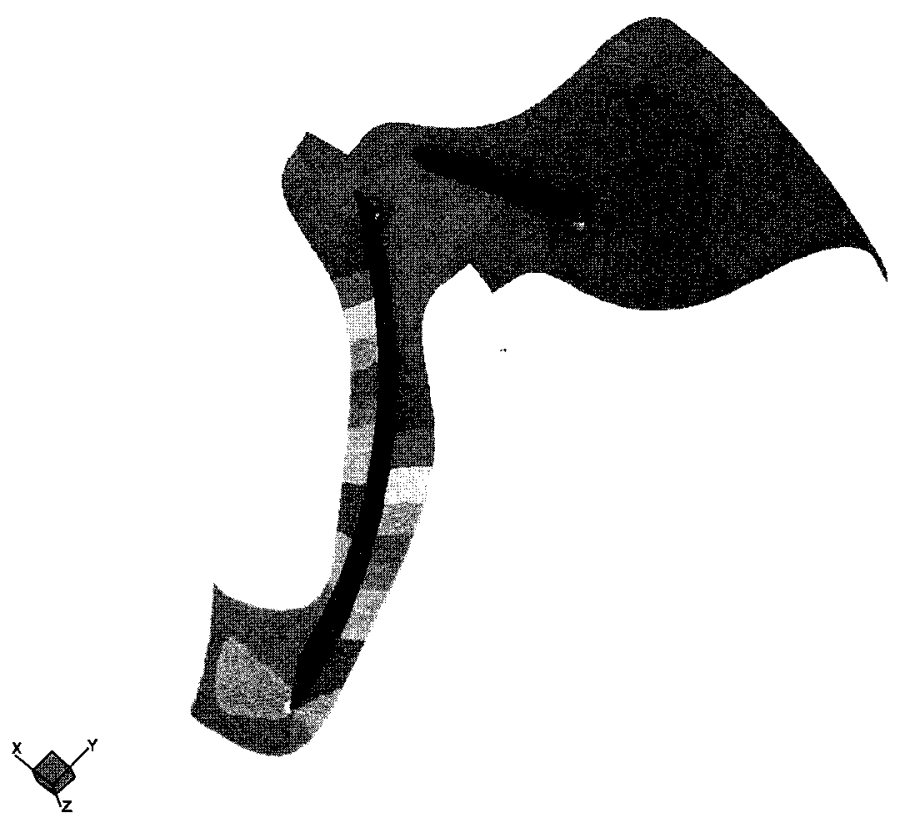

FIGURE 8 Static pressure field in the impeller and LSD at design flow, $\Phi=0.0275$, new design, mid-span (See Colour Plate at back of issue.).
A local separation zone occurred at the hub-suction side of the LSD vane near the trailing edge that results in a wake flow region in the return bend entrance.

At overload (120\% design mass flow), impeller flow was without separation, but flow with a higher relative Mach number are found at the inlet, both on the suction and pressure sides of the blade. The latter is due to incidence effects and the corresponding turning required around the leading edge. For this flow case the LSD region has a strong recirculation zone on the pressure side of the blade, and the resulting wake washes out only in the second half of the return bend.

For smaller stages ( $\Phi=0.0146$ and lower), the flow field in the impeller and diffuser looks similar to that in the parent stage, both at design and off-design points. The

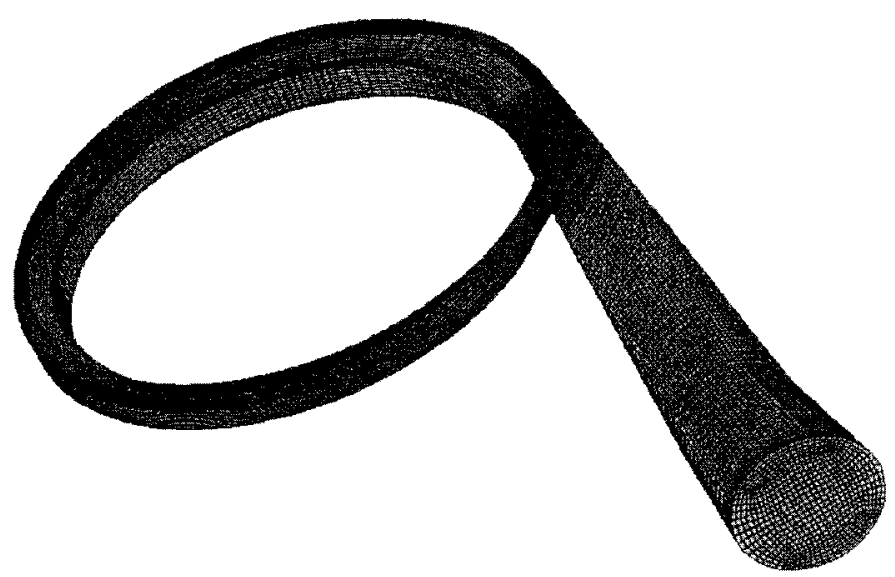

FIGURE 9 Volute grid for CFD model.

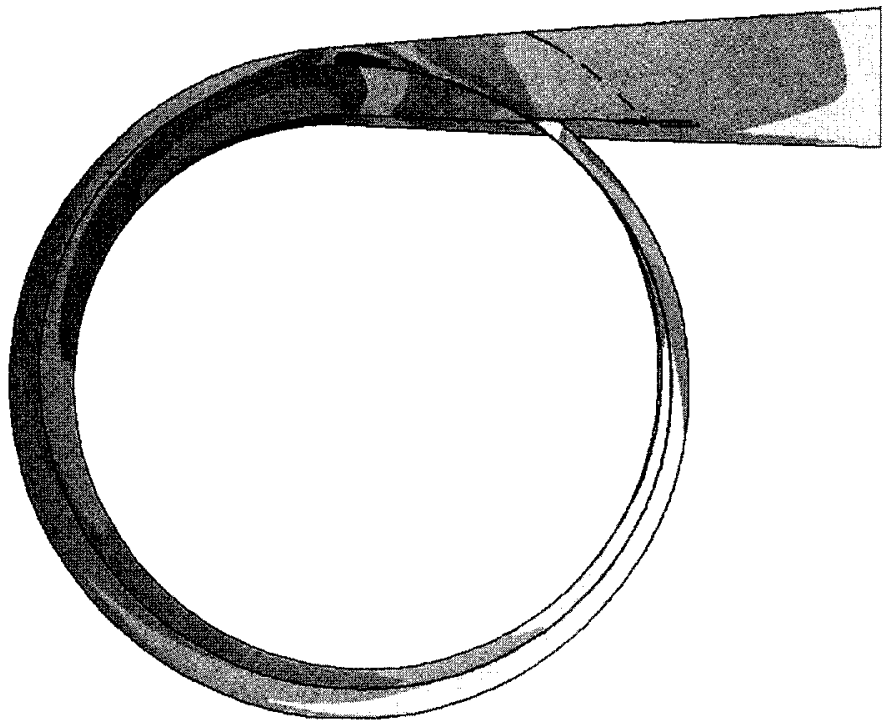

FIGURE 10 Volute static pressure contour plot (See Colour Plate at back of issue.). 
separation zone is found in the LSD region at the low flow condition on the suction side of the vane, close to the trailing edge and shroud surface. Figure 7 illustrates the flow field in the newly designed impeller with a flow coefficient of 0.0117. Static pressure plot in the impeller and the LSD mid-span region is shown in Figure 8.

At low flow, the recirculation eddy is washed out in the LSD quickly enough to smooth the velocity profile in the return bend. A typical low velocity zone in the impeller was located at the outlet on the suction side of the blade, becoming more severe toward the shroud surface. In addition, the wake entering the diffuser from the impeller is not very strong.
A CFD model was built to analyze the flow in the discharge volute. As shown in Figure 9, about 400,000 nodes were used to mesh the bend following the last stage impeller and diffuser, the volute, and the discharge nozzle. A commercial code Star-CD ${ }^{\mathbb{R}}$ from Computational Dynamics Ltd. was utilized to run this model. A mass flow rate boundary condition was applied to the inlet, at the entrance to the bend. At the exit of the discharge nozzle, an outflow boundary condition was applied. Figure 10 shows the static pressure distribution computed by the CFD model. The flow field in the volute was found uniform with no major loss regions.

Presently Dresser-Rand routinely utilizes coupled fully 360 degrees geometric CFD grids to model steady-state and
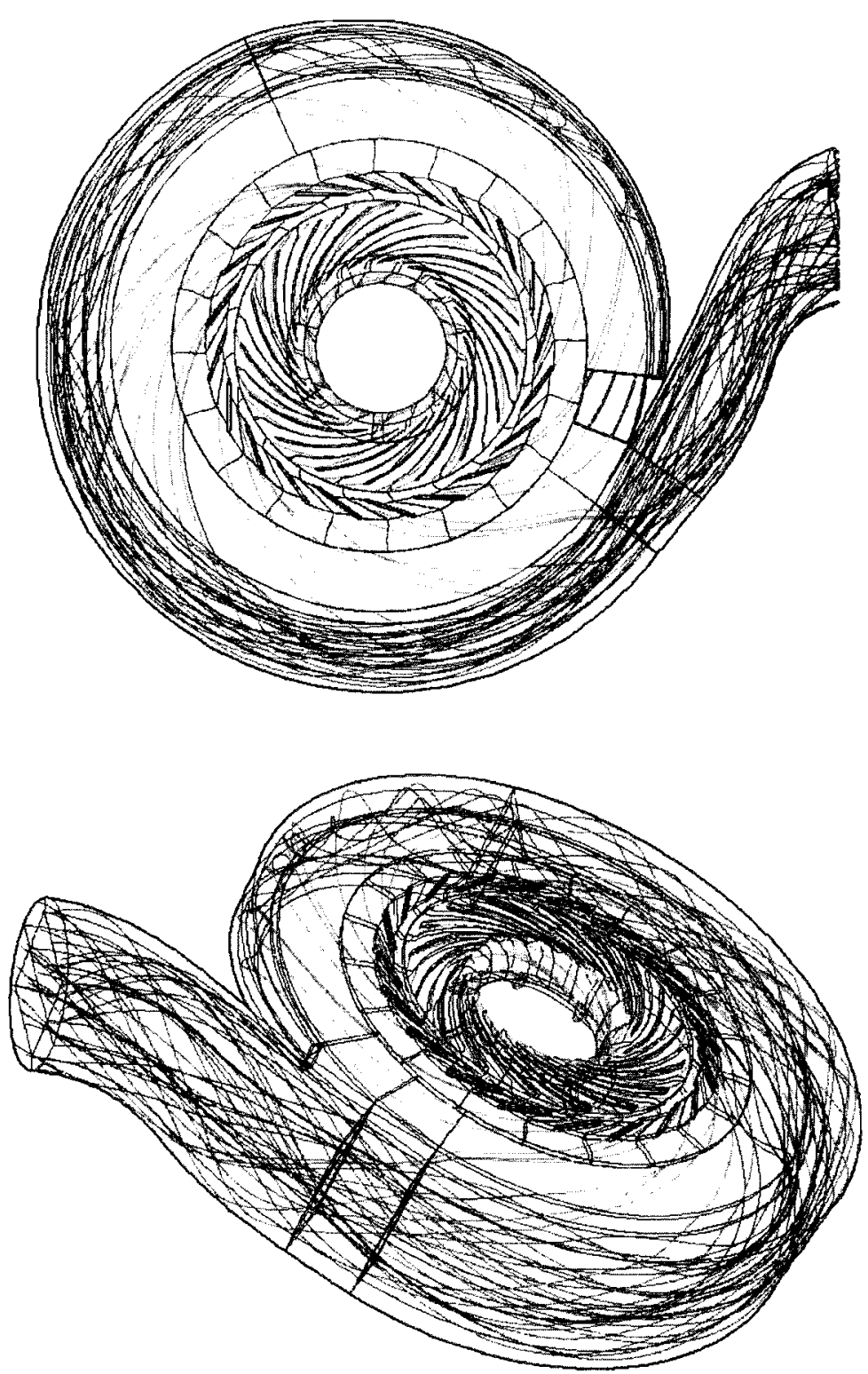

FIGURE 11 Streak lines in $360^{\circ}$ coupled CFD model of last stage of low flow compressor (See Colour Plate at back of issue.). 
transient flows in centrifugal compressor stages, especially those including non-axisymmetric geometric components as inlets, volutes and sidestreams (Biba et al., 2000). To accelerate time-consuming grid generation work for volutes a proprietary parametric tool has been developed (Soni, 2000). An example of the CFD solution in the last stage of high-pressure compressor is shown in Figure 11. The model containing low flow impeller, LSD, and volute was used to determine the impact of volute design on the performance and operating range of low flow stage.

\section{PERFORMANCE COMPARISON}

Figure 12 shows peak efficiency and polytropic head coefficient at peak efficiency based on the CFD results and Single Stage Test Rig (SSTR). The first item that should be noted is that CFD overpredicts the test data for low flow coefficients, while underpredicting data at higher values of flow coefficients. The underlying causes for these differences are most likely due to influences external to the main flow path which were not modeled (e.g., friction in the impeller cavity and leakage) as well as numerical sources (e.g., grid dependency) and turbulence modeling. There are several other items of interest that should be noted from Figure 12. First the impeller efficiency curves predicted by CFD track relatively closely to one another, although the new impeller appears to exhibit slightly lower efficiency in the lower flow capacity stages. On a stage basis the new
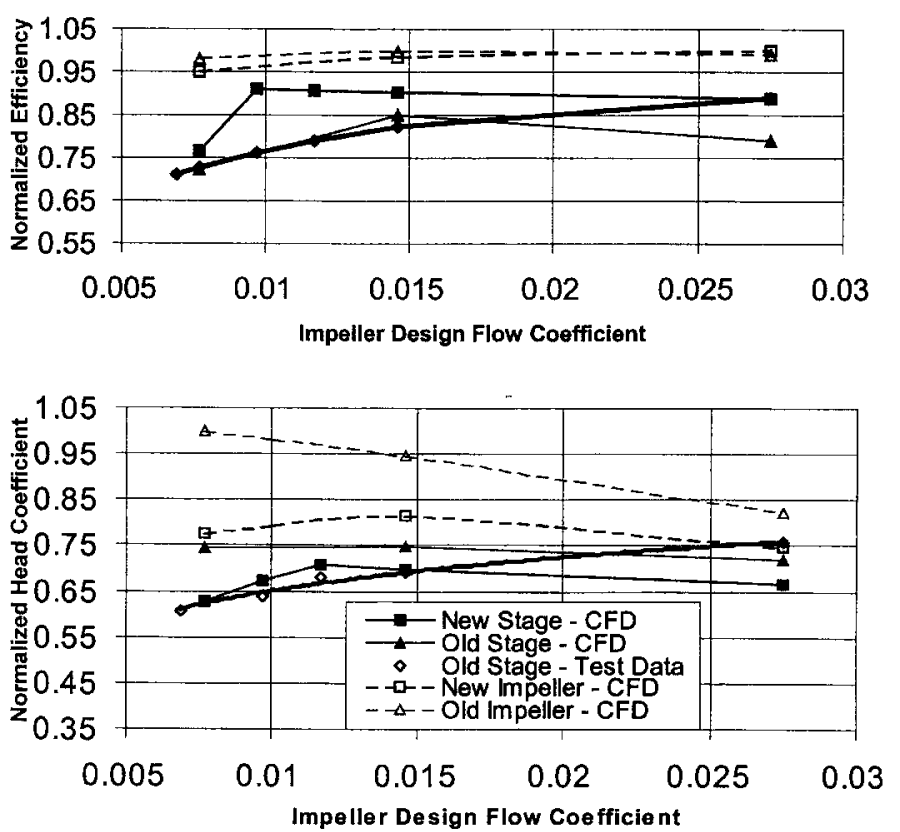

FIGURE 12 Comparison of normalized optimum efficiency and head coefficient at best efficiency point for new and old design impellers and stages. stages appear to exhibit a near constant peak efficiency across almost the entire flow range. The older stage peak efficiencies follow more closely to the empirical curve.

An alternative presentation of the peak efficiency and head coefficient at peak efficiency is shown in Figure 13. The CFD data shown on this plot are normalized by maximum values of peak efficiency and head coefficient, while the empirical data was normalized to agree with the CFD results for the old stage with $\Phi=0.0275$. These results demonstrate more clearly the differences in curve shape and relative performance of the stage designs. Clearly the new stage shows significant improvement in performance when compared to the old stage. The actual level of improvement cannot be determined without further calibration of the predictions with empirical data.

Head coefficient $\mu$ curves shown in Figures 12 and 13 correspond to the peak efficiency operating points. The results indicate higher head levels at peak efficiency for both the current ("old") impeller and stage designs. The results also imply better pressure recovery in the stationary components for the new design, based on the comparison of $\mu$ for the impeller and stage curves.

The general shape of the CFD predicted curves for $\mu$ are relatively flat with a peak in the $0.010-0.015$ flow coefficient range, while the empirical curve and SSTR data suggest an increasing trend for $\mu$ with flow coefficient. The reason for this discrepancy most likely lies in the lack of leakage and external secondary flow path windage losses; internal flow path roughness may also be a factor. In addition, previous comparison with SSTR data indicates that the CFD results appear to underestimate the impeller slip factor.
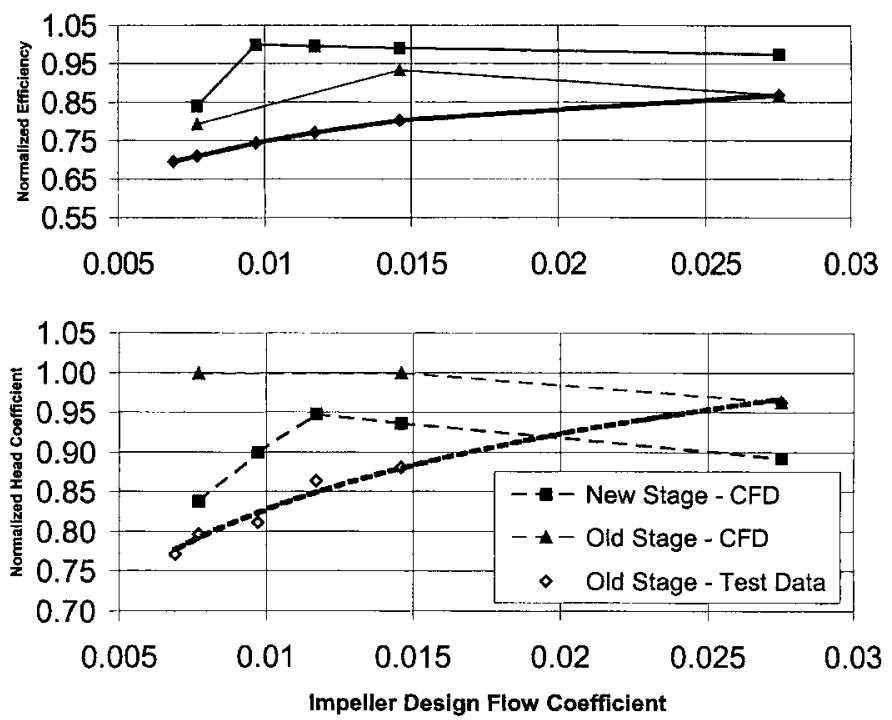

FIGURE 13 Comparison of normalized optimum efficiency and head coefficient at best efficiency point for new and old design stages. Alternative normalization. 


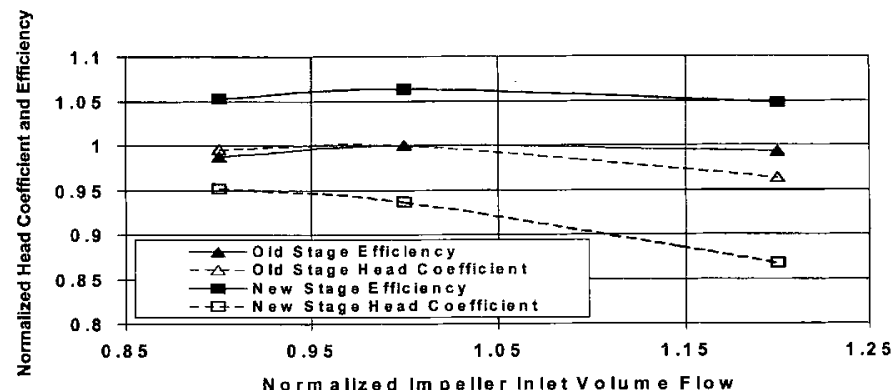

FIGURE 14 Comparison of CFD-based performance curves for new and old stages at $\Phi=0.0146$.

Figure 14 illustrates individual stage performance along a speed line. The new stage with $\Phi=0.0146$ exhibits the typical curve shape expected with LSD's, higher peak efficiency with slightly shorter range.

Relative peak efficiency adjustments for STGPERF were suggested based on the CFD results obtained assuming that the difference in head and efficiency between the new stage design and the original design may be identical to the actual change in performance of the physical machine.

\section{COMPARISON WITH PRODUCTION TEST}

The one-dimensional analysis code STGPERF was modified based on the aforementioned CFD results to ensure performance prediction for stages with newly designed low flow impellers. Based on stage-by-stage calculations the overall compressor performance was predicted at test conditions and compared with test results. Intermediate test data were gathered between stages where total pressure and temperature probes were installed in the return bend area.

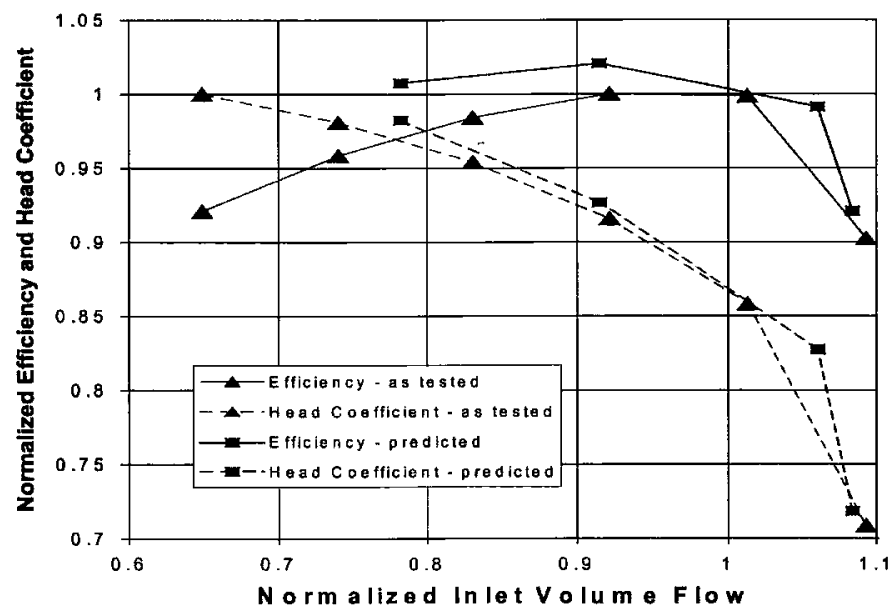

FIGURE 15 Comparison between tested and predicted overall compressor performance characteristics.
Comparison of newly predicted and tested data for the entire compressor is shown in Figure 15. The results demonstrate very good agreement of head coefficient and relatively good agreement of efficiency values. The discrepancy does not exceed $2.5 \%$ and is greater at low flow capacity conditions.

\section{CONCLUSIONS}

The proposed new stage design demonstrated improved efficiency compared to the previous design that made new geometric configuration successful in a high pressure compressor and for similar applications. It should be noted that when applying the newly designed low flow stages, operating range is reduced due to utilization of LSD's. Additional design improvements are possible, e.g., adjusting impeller leading edge position and/or changing the number of blades, optimizing return channel/bend geometry, etc.

The efficiency improvement has been predicted by a series of CFD calculations at various flow capacity values and verified experimentally by testing the compressor with the newly designed changes. Qualitative agreement between computational and test data has been obtained. Quantitatively, the CFD calculations slightly over-predict the performance. This discrepancy is caused by numerous factors not being taken into account in the computational model and CFD tools; such as secondary losses in labyrinth seal passages, surface roughness, inlet and intermediate flow non-uniformity, transient effects, turbulence modeling, near-wall flow resolution, etc. Some of these effects represent a challenge for CFD methods to predict them accurately. At its current level of development CFD can not be used for direct performance predictions. However, CFD is an excellent tool for providing data for relative dimensionless comparison and analysis of the impact of design and flow parameters on performance of centrifugal compressor components.

For practical reasons, it is possible to define a set of key parameters that will allow a designer to perform a parametric study of the impact on stage performance using CFD. Results of that study, together with available test data, can be used to tune the existing models implemented in a semi-empirical 1D code (like Dresser-Rand's STGPERF) to enable it to predict stage and overall compressor performance with accuracy. Ultimately, this parametric approach will also make the $1 \mathrm{D}$ code more generic in nature (i.e., able to analyze the full range of stage geometry) as well as open the doors to if's application as an inverse design tool that could be used to tailor stage design to operating specific conditions.

In current conditions of rising research and production test costs, the problem of calibration of CFD codes for 
turbomachinery becomes a key factor in performance prediction. Consequently, Dresser-Rand continuously strives to develop its test and computational programs, including both SSTR rigs and expanding CFD capabilities.

\section{Acknowledgments}

The authors would like to thank Dresser-Rand Company for the permission to publish this work. We are grateful all colleagues at Core Technology Department of DresserRand Olean Operations for their contributions into the results and for useful discussions. This article is a revised version of the paper presented at the $8^{\text {th }}$ International Symposium on Transport Phenomena and Dynamics of Rotating Machinery, March 26-30, 2000, Honolulu, Hawaii. ${ }^{1}$

\section{NOMENCLATURE}

$\mathrm{D}_{2}$ overall impeller diameter

$\mathrm{D}_{5}$ diffuser outlet diameter

$\mathrm{Q}_{0}$ volumetric flow at stage inlet

$\mathrm{u}_{2}$ impeller speed at overall diameter

$\Phi$ flow coefficient, $\Phi=4 \mathrm{Q}_{0} /\left(\pi \mathrm{u}_{2} \mathrm{D}_{2}^{2}\right)$ $\eta \quad$ polytropic efficiency

$\mu$ polytropic head coefficient

\section{REFERENCES}

Balje, O. E. (1981) Turbomachines-Guide to Design, Selection and Theory, Wiley, New York.

Biba, Y. I., Liu, Z. and Hill, D. L. (2000) Pipeline Compressor Redesign with the Consideration of Both Noise and Performance, Proceedings of the International Pipeline Conference, October 1-5, Calgary, Canada, pp. 1317-1324. ASME IPC-00-0164.

Casey, M. W., Dalbert, P. and Schurter, E. (1990) Radial Compressor Stages for Low Flow Coefficients, Presented at the 4th European Congress on Fluid Machinery for Oil, Petrochemical and Related Industries, The Hague, The Netherlands, IMech Paper C403/004.

Dalbert, P., Ribi, B., Kmeci, T. and Casey, M. V. (1999) Radial Compressor Design for Industrial Compressors, Proceedings of the Institution of Mechanical Engineers, Part C: Journal of Mechanical Engineering Science, 213(1), 71-83.

Soni, B. K., Hur, J. and Collins, E. M. et al. (2000) Parametric Grid Generation Applicable to Design Optimization of Centrifugal Compressor Configurations, Presented at the 8th AIAA/NASA/USAF/ ISSMO Symposium on Multidisciplinary Analysis and Optimization, September 6-8, Long Beach, CA. AIAA Paper 2000-4862.

Sorokes, J. M. and Koch, J. M. (1996) The Use of Single and Multi-Stage Test Vehicle in the Development of the Dresser-Rand DATUM Compressor, Dresser-Rand Technology Journal, 2, 132-147.

Sorokes, J. M. and Welch, J. P. (1992) Experimental Results on Rotatable Low Solidity Vaned Diffuser, Presented at the IGTI Gas Turbine Aeroengine Congress and Exposition, Cologne, Germany, ASME Paper 92-GT-19.

\footnotetext{
'(C) 2000 PCTFE (Pacific Center of Thermal-Fluids Engineering).
} 

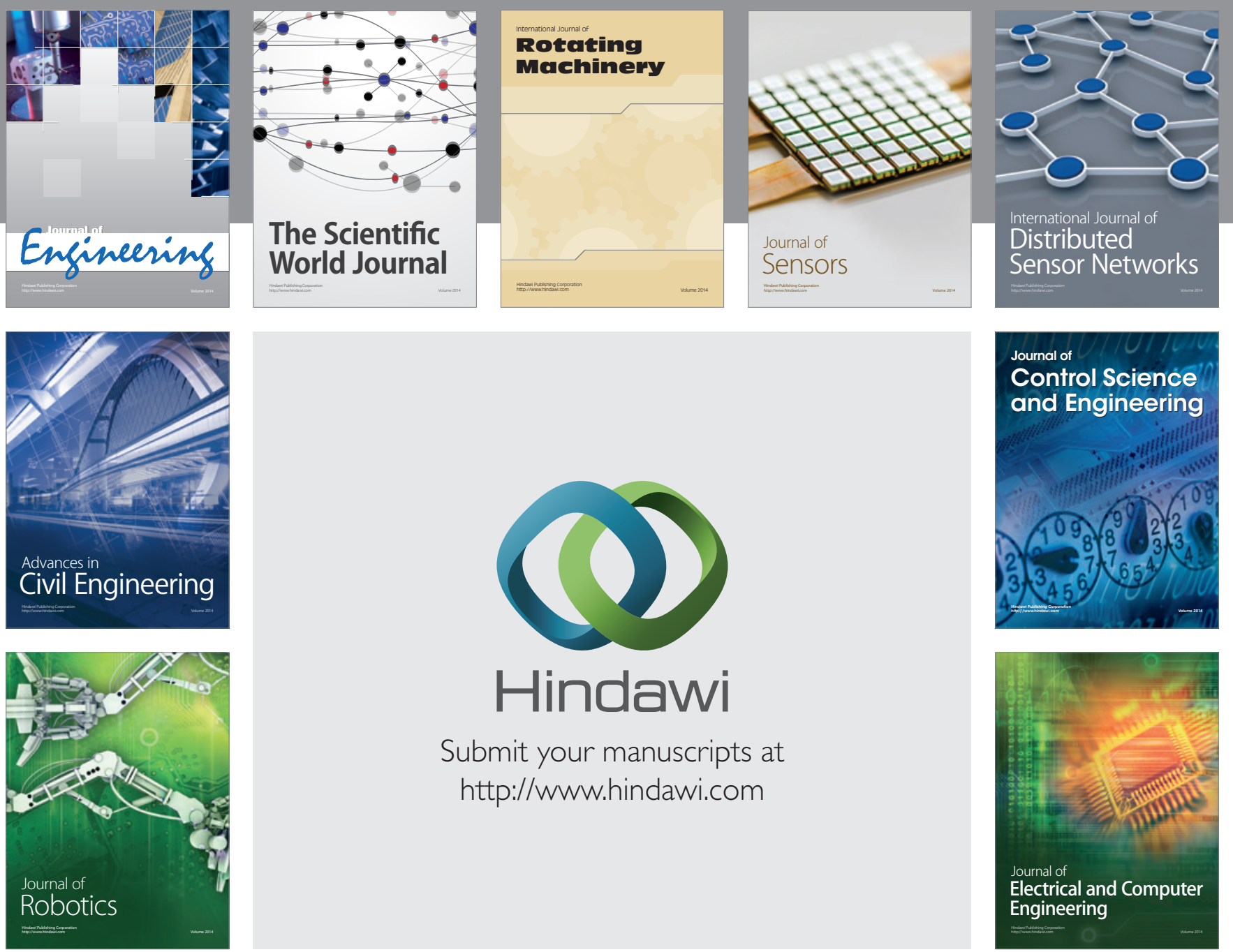

Submit your manuscripts at

http://www.hindawi.com
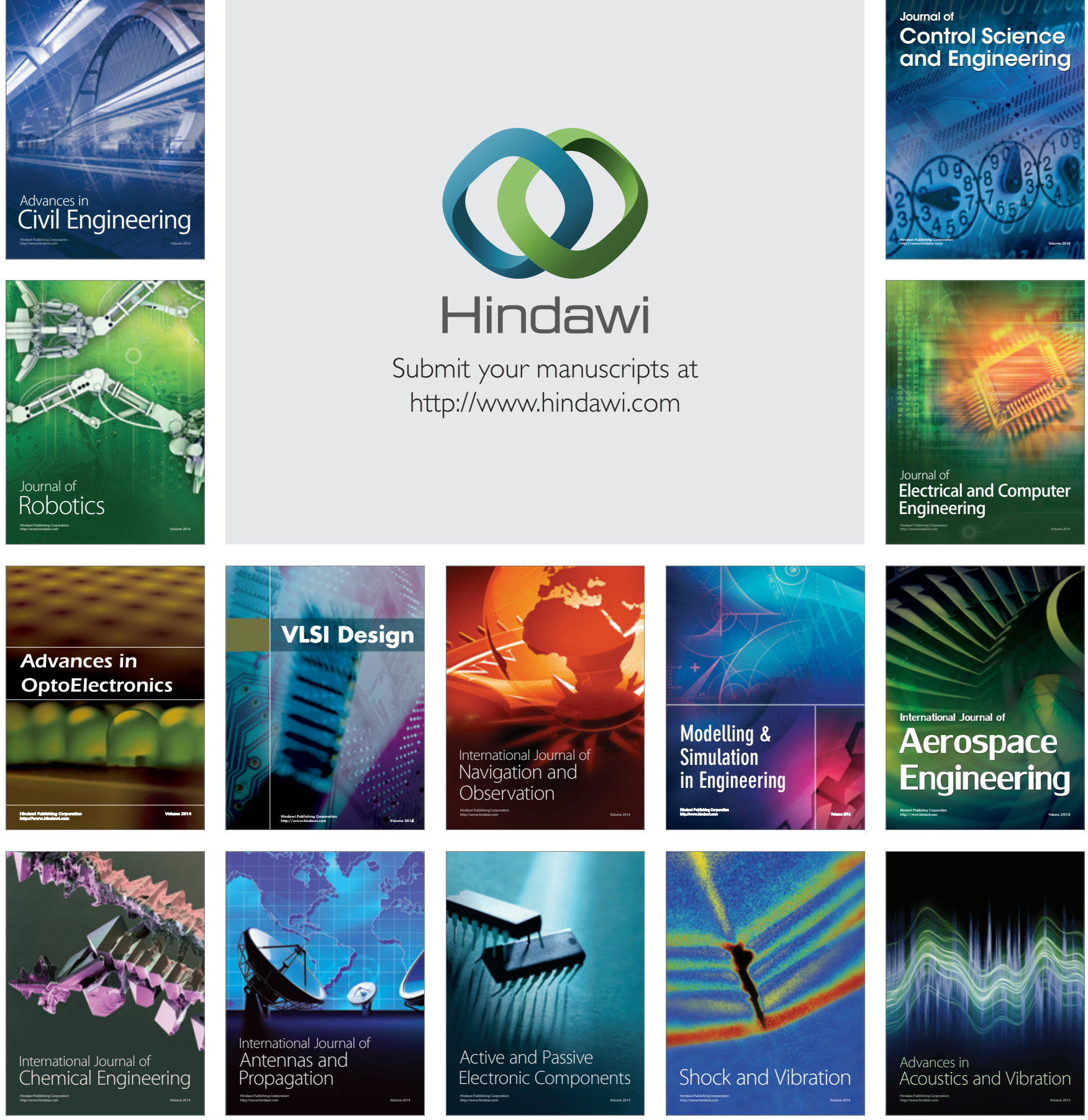